\title{
THE SCATTER FOCUSSING OF MULTI-GeV CHARGED PARTICLES AND NEUTRAL HADRONS*
}

\author{
LAWRENCE W. JONES and DONALD G. KOCH \\ Randall Laboratory of Physics, University of Michigan, Ann Arbor, Michigan 48104, U.S.A.
}

Received 16 July 1973

The multiple Coulomb scattering of charged particles and the nuclear elastic scattering of neutral hadrons may serve as a basis for the design of lenses for beams of such particles. The

\section{Introduction}

Charged particles may be focussed by multiple scattering in a suitably designed lens of scattering material. Similarly, neutral hadrons may be focussed by elastic scattering from nuclei using a specially constructed lens.

These notions and some experımental observations were originally set forth in papers by one of us in 1964 on The random optics of particle beams ${ }^{1}$ ). In this paper, we present a more complete - and more correct - formulation of the multiple-scattering focussing problem, with numerical examples. We then present a quantitative study of the elastic scattering lens for neutrons (or other neutral hadrons) noting possible applicatıons and numerical examples.

The history of this interest began in 1963 when setting up experiments in the extracted proton beam of the Brookhaven Cosmotron. Polaroid film constituted a convenient detector technique for aligning and focussing the beam. In order to locate a reference point or line on the film, a metal object was placed at a known position ahead of the film and its shadow was recorded on film. The curious properties of these shadows, or (more properly) images, was the starting point for the ideas discussed below. If the film is placed against an object, no image is seen, but if the film is a few $\mathrm{cm}$ beyond the object, the image is surprisingly sharp and detarled. That no image is seen in the former case in reasonable, since the attenuation of a $3 \mathrm{GeV}$ proton beam $1 \mathrm{n} \frac{1}{8}$ " of brass is only about $2 \%$. The image that develops with spacing is then due to multiple Coulomb scattering in the metal.

In order to understand such images, consider a Cartesian coordinate system with a parallel beam in the $+z$ direction and a scattering slab at $z=0$ extendIng over all $y$ and all positive $x(x \geq 0)$ with open space

* Work supported by the U S National Scrence Foundation. optical and spectral properties of such lenses are explored and numerical examples are given.

at all negative $x$. Neglecting interactions in the slab, the beam flux at $x \gg 0$ and at $x \ll 0$ for positive values of $z$ is unaffected by the slab, since as much flux is scattered into a given area as out of one. On the other hand, for values of $x$ near $|x| / z \approx \theta_{\text {rms }}$ (where $\theta_{\text {rms }}$ is the multiple Coulomb scattering angle), the intensity will be reduced for $x$ positive, since some particles are scattered out of that region, and the intensity will be

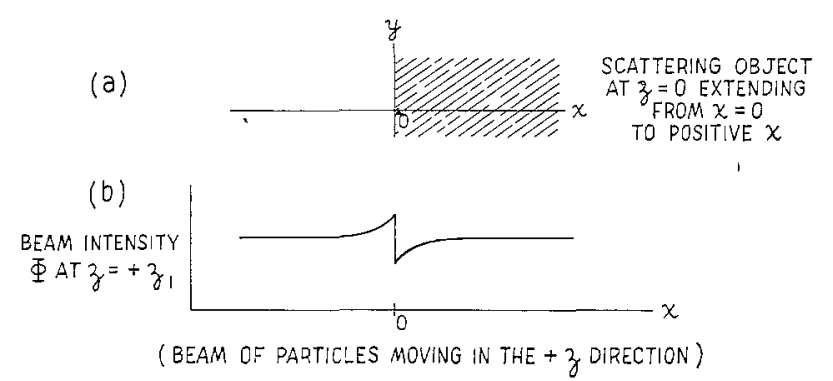

Fig. 1 The nature of an image resulting from a semi-infinite slab of scattering material. (a) The scatterer, at $z=0$; (b) the particle flux $\Phi$ vs $x$ at $z_{1}>0$.

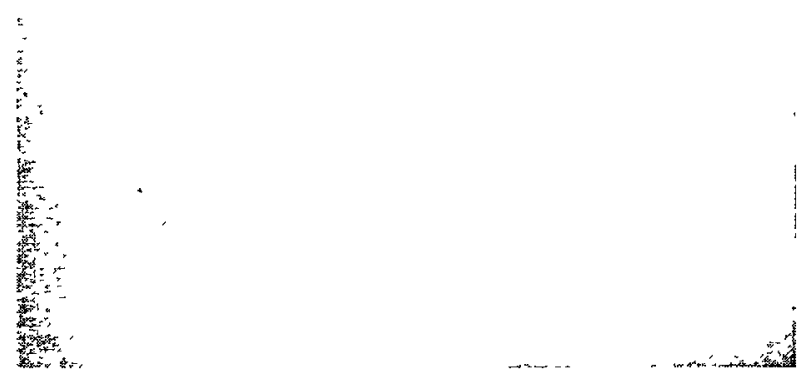

Fig. 2. Scatter images of cylinders of lead (left) and aluminum (right) in a proton beam of $3 \mathrm{GeV}$. Each cylinder was $1^{\prime \prime}$ in diameter and 1 " in length. The film was $15^{\prime \prime}$ beyond the cylunders. The images are positive prints, i.e., lighter shades are higher intensity. 
increased for $x$ negative since no particles are scattered out but some are scattered in. This is illustrated in fig. 1 . In fig. 2 we have reproduced the image of two $1^{\prime \prime}$ diameter, $1^{\prime \prime}$ long cylinders (axes parallel to the proton beam), one of lead and one of aluminum. The images are positive prints, so that bright areas correspond to higher proton fluxes. That more subtle image detail may be visualized is seen in figs. 3 and 4, where a wrist watch and an electric hand drill are imaged.

\section{A multiple Coulomb scattering lens}

The notion that multiple Coulomb scattering can be used to enhance the flux of charged-particle beam particles at some region downstream of a scatterer suggests that it should be possible to so arrange

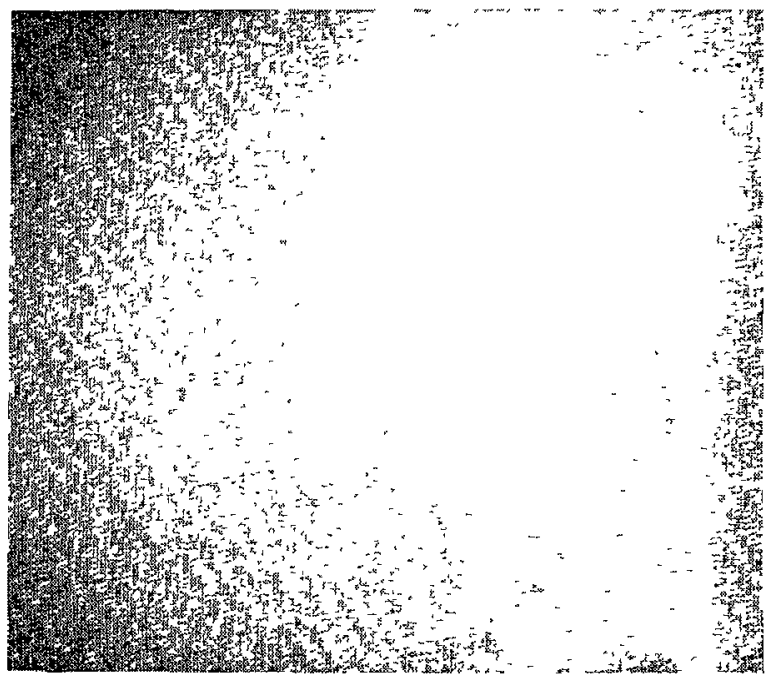

Fig. 3. A wrist watch imaged by scattering of $3 \mathrm{GeV}$ protons.

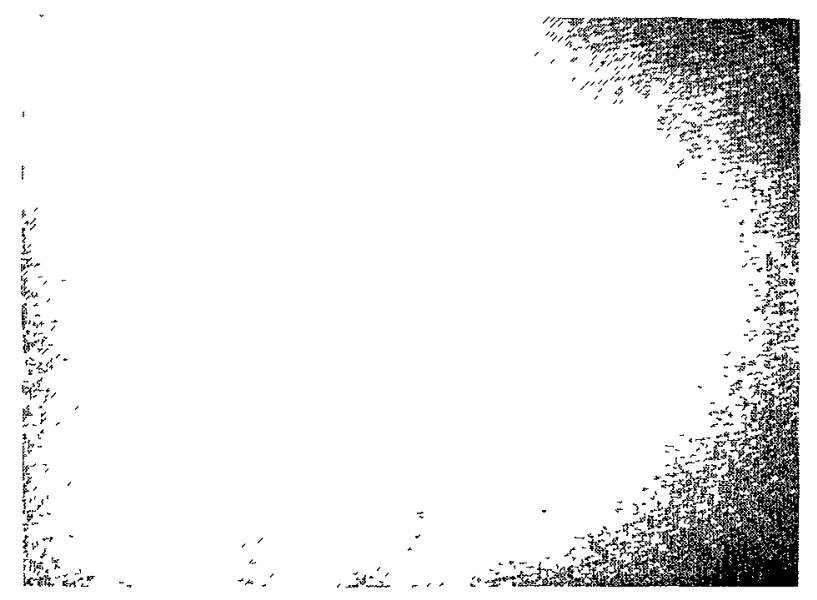

Fig. 4. A portion of an electric hand drull imaged with $3 \mathrm{GeV}$ protons. scatterers as to maximize this flux enhancement, i.e., to design a "lens". We may proceed below with such a lens design, making use of simplified expressions for multiple Coulomb scattering ${ }^{2}$ ).

The probability of scattering through a projected angle $\theta_{x}$ upon traversal of a scattering material of thickness $l$ is given by

$$
P\left(\theta_{x}\right) \mathrm{d} \theta_{x}=\frac{1}{\theta_{0}}\left(\frac{2}{\pi}\right)^{\frac{1}{2}} \exp -\left[\theta_{x}^{2} / 2 \theta_{0}^{2}\right] \mathrm{d} \theta_{x}
$$

where

$$
\theta_{0}=\frac{15.0}{p v}\left(l / I_{\mathrm{R}}\right)^{\frac{1}{2}}=\left(\theta_{x}\right)_{\mathrm{rms}},
$$

with $p r$ in $\mathrm{MeV}$ and $l_{\mathrm{R}}$ the radiation length in the material. The probability of scattering through a space angle $\theta$ is then

$$
P(\theta) \mathrm{d} \theta=\frac{\theta}{\theta_{0}^{2}} \exp -\left[\theta^{2} / 2 \theta_{0}^{2}\right] \mathrm{d} \theta .
$$

where

$$
(\theta)_{\mathrm{rms}}=\sqrt{2}\left(\theta_{x}\right)_{\mathrm{rms}}=\frac{21.2}{p v}\left(l / l_{\mathrm{R}}\right)^{\frac{1}{2}} .
$$

In these expressions and throughout we will use only the small angle approximation where $\theta \cong \sin \theta \cong \tan \theta$. The probability of scattering into a solid angle $\mathrm{d} \Omega$ at $\theta$ is then

$$
\frac{P(\theta)}{2 \pi \theta} \theta \mathrm{d} \theta \mathrm{d} \varphi=\frac{P(\theta)}{2 \pi \theta} \mathrm{d} \Omega .
$$

Now consider a uniform parallel flux $\Phi_{0}$ of particles (of $p, v$ ) incident on a scattering slab of thickness $l$ at $z=0$ as in fig. 5. At $r$ from the $z$ axis the number of particles incident on a small area $\mathrm{d} A_{0}$ will contribute to the flux at $z=d, r=0$ an amount $\mathrm{d} \Phi$ given by

$$
\mathrm{d} \Phi=\Phi_{0} \mathrm{~d} A_{0}\left[\frac{P(\theta)}{2 \pi \theta}\right] \frac{\mathrm{d} \Omega}{\mathrm{d} A_{d}},
$$

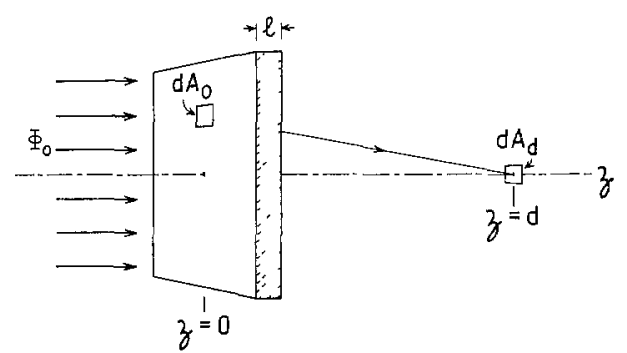

Fig. 5. Scattering of a parallel beam by a plane slab of thickness $l$. 
where $\mathrm{d} A_{d}$ is an element of area normal to the $z$ axis at $z=d$. Then, in the small angle approximation,

$$
\begin{aligned}
& \mathrm{d} \Omega \cong \mathrm{d} A_{d} / d^{2}, \\
& r / d \cong \theta,
\end{aligned}
$$

and

$$
\mathrm{d} A_{0}=r \mathrm{~d} r \mathrm{~d} \varphi
$$

Therefore,

$$
\mathrm{d} \Phi=\Phi_{0} \frac{\mathrm{d} \varphi}{2 \pi} P(\theta) \mathrm{d} \theta
$$

Integratıng around an annulus of radius $r$ and width $\mathrm{d} r$ over $\varphi$ from 0 to $2 \pi$ gives

$$
\mathrm{d} \Phi=\Phi_{0} P(\theta) \mathrm{d} \theta .
$$

If we now use a scatterer in the shape of a disc of inner radius $r_{1}$ and outer radius $r_{2}$, subtending angles from the "focal" plane $(z=d, r \cong 0) \theta_{1} \cong r_{1} / d, \theta_{2} \cong r_{2} / d$, the total flux $\Phi_{\mathrm{T}}$ in this focal plane is given by

$$
\Phi_{\mathrm{T}}=\Phi_{0}\left[1+\int_{\theta_{1}}^{\theta_{2}} P(\theta) \mathrm{d} \theta\right]>\Phi_{0},
$$

so that some net gain in flux is achieved.

One can do better than use a untform slab, of course. The scatterer may be contoured in the form of a paraboloid of revolution such that

$$
\theta=\alpha \theta_{0},
$$

or

$$
\frac{r}{d}=\frac{15 \alpha}{p \iota}\left(l / l_{\mathrm{R}}\right)^{\frac{1}{2}}
$$

so that $l \propto r^{2}$.

Now

$$
\Phi_{\mathrm{T}}=\Phi\left[1+\int_{\theta_{1}}^{\theta_{2}} \frac{\alpha^{2}}{\theta} \exp \left(-\frac{1}{2} \alpha^{2}\right) \mathrm{d} \theta_{0}\right],
$$

or

$$
\Phi_{\mathrm{T}}=\Phi\left[1+\alpha^{2} \exp \left(-\frac{1}{2} \alpha^{2}\right) \ln \left(\theta_{2} / \theta_{1}\right)\right] .
$$

For fixed $\theta_{1}$ and $\theta_{2}, \Phi_{\mathrm{T}}$ is maximum for $\alpha=\sqrt{2}$, or

$$
\begin{aligned}
& \Phi_{\mathbf{T}} / \Phi_{0}=\left[1+\frac{2}{\mathrm{e}} \ln \left(\theta_{2} / \theta_{1}\right)\right] \\
& \Phi_{\mathrm{T}} / \Phi_{0}=\left[1+0.7358 \ln \left(\theta_{2} / \theta_{1}\right)\right] .
\end{aligned}
$$

This geometry is illustrated in fig. 6 .

The spectral properties of the lens, or chromatic aberration, can be found by study of eq. (8), which we may rewrite here as

$$
\mathrm{d} \Phi(p)=\Phi_{0}(p) \frac{\theta \mathrm{d} \theta}{\theta_{p}^{2}} \exp -\left[\theta^{2} / 2 \theta_{p}^{2}\right],
$$

where $\theta_{p}$ is the r.m.s. scattering angle for particles of momentum $p$ and $\Phi(p)$ is the flux of those particles. From eq. (2),

$$
p \theta_{p}=p_{0} \theta_{0}
$$

and from eqs. (12) and (13),

$$
\theta=\theta_{0} \sqrt{ } 2
$$

so that

$$
\theta_{p}=\frac{p_{0} \theta}{p \sqrt{ } / 2}
$$

Consequently

$$
\mathrm{d} \Phi(p)=\Phi_{0}(p) 2\left(\frac{p}{p_{0}}\right)^{2} \exp -\left[p^{2} / p_{0}^{2}\right] \frac{\mathrm{d} \theta}{\theta},
$$

and

$$
\Phi(p)=\Phi_{0}(p) 2\left(\frac{p}{p_{0}}\right)^{2} \exp -\left[p^{2} / p_{0}^{2}\right] \ln \left(\frac{\theta_{2}}{\theta_{1}}\right) .
$$

The ratio of the flux at $p$ relative to the flux at $p_{0}$, the design momentum, can then be found relative to the incident flux at each momentum. This is:

$$
\frac{\Phi(p) / \Phi_{0}(p)}{\Phi\left(p_{0}\right) / \Phi_{0}\left(p_{0}\right)}=\left(\frac{p}{p_{0}}\right)^{2} \exp \left[1-\left(p^{2} / p_{0}^{2}\right)\right] .
$$

As before, $\Phi_{\mathrm{T}}=\Phi+\Phi_{0}$, however it is convenient to leave eq. (18) in this form as it is independent of $\theta_{2} / \theta_{1}$. The response function of eq. (18) is plotted in fig. 7 .

Consider a numerical example of such a scatter focussing lens where $\theta_{2} / \theta_{1}=20$. Here, for the design

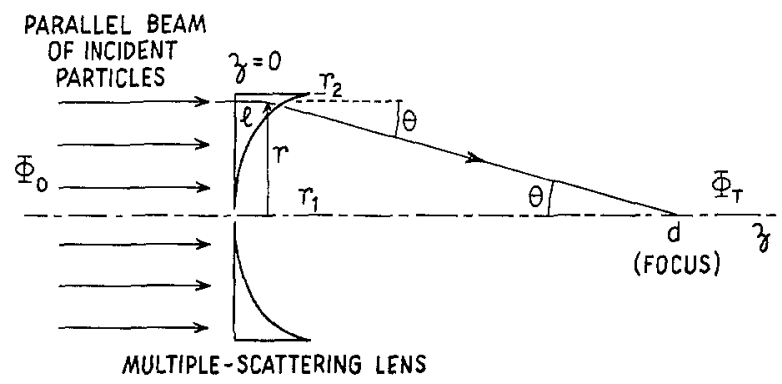

Fig. 6. The geometry of a multıple scattering lens, with a thrck. ness $l \propto r^{2}$ from $r_{1}$ to $r 2$. 
momentum $p$,

$$
\Phi_{\mathrm{T}} / \Phi_{0}=3.204 \text {. }
$$

This might in practice correspond to a lens of $20 \mathrm{~cm}$ outer diameter with a $1 \mathrm{~cm}$ diameter hole in the center. Thus could be a lead lens of $20 \mathrm{~cm}$ diameter and $1 / 5$ of a nuclear interaction mean free path thick at the edge. Since the interaction mean free path in lead is about $14 \mathrm{~cm}$, it would be $2.8 \mathrm{~cm}$ thick at $r=10 \mathrm{~cm}$. As $l_{\mathrm{R}}=05 \mathrm{lcm}$ for lead, the focal length would then be found from

$$
r / f=r / d=\theta_{2}=\frac{15}{p v} \sqrt{ } /(2.8 / 0.51)=\frac{35.147}{p v}
$$

The focal length is then given by

$$
f=\frac{p v r_{2}}{15.0}\left(l_{\mathbf{R}} / l_{2}\right)^{\frac{1}{2}}
$$

which, for this numerical example, gives

$$
f=0.2845(p u) \text {. }
$$

where $p \iota$ is in MeV and $f$ is in $\mathrm{cm}$. Thus for $p \iota=10 \mathrm{GeV}$ $f=28.45 \mathrm{~m}$.

This lens would enhance the axial flux over the central $1 \mathrm{~cm}$ diameter spot by a factor of 3.2 , and with a focal length altogether reasonable. Such a lens is obviously very much less costly than a magnetic lens (quadrupole pair, etc.), it is compact and trouble-free. As such it could be totally buried in shielding, or could be used in an intense radiation environment. On the other hand, the gain through scatter focussing should not be misunderstood Our factor of 3.2 should properly be compared with a factor of 400 for a properly designed magnetic lens of the same aperture.

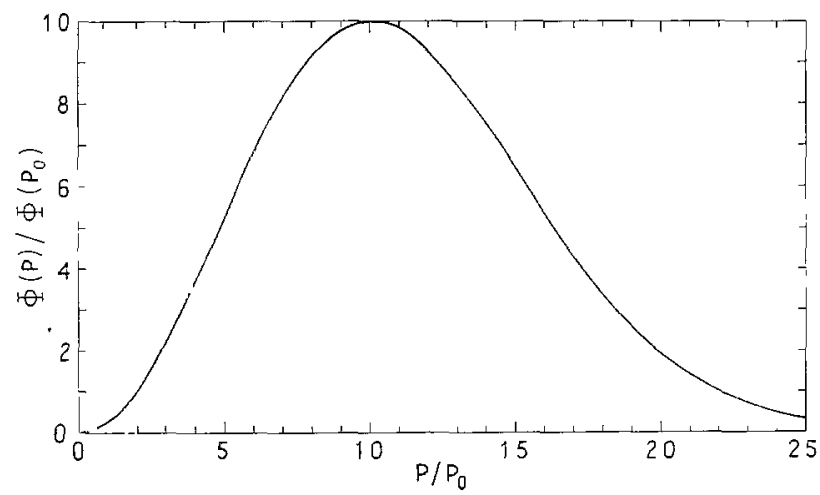

Fig. 7. The spectral enhancement of the "focussed" flux $\Phi$ in the focal plane of a scatterng lens. The expression for $\Phi(p)$ / $\Phi\left(p_{0}\right)$ is from eq. (18), with the assumption $\Phi_{0}(p)=\Phi_{0}\left(p_{0}\right)$.
In fact the gains in scatter focussing are proportional to ln (aperture) while the gans in proper geometrical optics are proportional to (aperture) ${ }^{2}$.

\section{Elastic scatter focussing of neatral hadrons}

\subsection{GeNerAL PRINCIPLES}

The situation is somewhat more interesting in the case of neutral hadrons where no other focussing is possible. Here nuclear diffraction elastic scattering plays a role exactly analogous to multiple Coulomb scatterıng (section 2) except that atomic mass number (nuclear radius) must be used as variable instead of scattering thickness, and scattering must compete with nuclear absorption (Inelastic scattering) so that the gains are much more limited. The point 1s, of course, that no other means exist for performing equivalent operations. Further, the chromatic aberration may be used to bias a continuous energy spectrum to favor pariticular energies.

Our attention is directed primarily toward energies above a GeV. We will use neutrons for our numerical examples, although equivalent expressions almost certainly hold for $\mathrm{K}_{0}^{\mathrm{L}}$ beams ${ }^{3}$ ). The expressions which play the role of eqs. (1)-(5) in the neutron case are given below, where the approximate analytic expressions are taken from the data of Parker et l. $^{4}$ ) and Ringia et al. ${ }^{5}$ ) for $4-5 \mathrm{GeV}$ neutrons. The elastic scattering is presumed here to be completely imaginary and due entirely to optical diffraction. In this case, then

$\frac{\mathrm{d} \sigma}{\mathrm{d}|t|}=\frac{\sigma_{\mathrm{T}}^{2}}{16 \pi \hbar^{2}} \exp (-B|t|)=\frac{\sigma_{\mathrm{T}}^{2}}{16 \pi \hbar^{2}} \exp \left(-B p^{2} \theta^{2}\right)$

and since

$$
\begin{gathered}
\frac{\mathrm{d} \sigma}{\mathrm{d} \Omega}=\frac{p^{2}}{\pi} \frac{\mathrm{d} \sigma}{\mathrm{d}|t|}, \\
\frac{\mathrm{d} \sigma}{\mathrm{d} \Omega}=\frac{p^{2} \sigma_{\mathrm{T}}^{2}}{(4 \pi \hbar)^{2}} \exp \left(-B p^{2} \theta^{2}\right),
\end{gathered}
$$

where $p$ is in $\mathrm{GeV} / c$. We will adopt the following approximate but very satisfactory dependences of $\sigma_{\mathrm{T}}$ and $B$ on atomic number $A$ :

$$
\begin{gathered}
\sigma_{\mathrm{T}}=80 A^{\frac{2}{3}} \mathrm{mb}=8 \times 10^{-26} A^{\frac{2}{3}} \mathrm{~cm}^{2}, \\
B=10 A^{\frac{2}{3}}(\mathrm{GeV} / \mathrm{c})^{-2} .
\end{gathered}
$$

If neutrons are incident on a slab of thickness $l$, the maximum number will emerge after experiencing a single scattering if the thickness is equal to the mean free path of neutrons in the material, i.e. $l=\lambda$, where 
$\lambda=A /\left(N \rho \sigma_{\mathrm{T}}\right)$, with $A$ the atomic number, $N$ Avogadro's number, $\rho$ the density, and $\sigma_{\mathrm{T}}$ the total cross section. The flux of single scattered neutrons $\Phi_{\mathrm{s}}$, is then given in terms of the incident flux, $\Phi_{0}$, by

$$
\Phi_{\mathrm{s}}=\Phi_{0} \frac{\sigma_{\mathrm{el}}}{\sigma_{\mathrm{T}}}\left(\frac{1}{\mathrm{e}}\right),
$$

where $\sigma_{\mathrm{e} 1}$ is the elastic scattering cross section.

From eqs. (22)-(24),

$$
\frac{\sigma_{\mathrm{e} 1}}{\sigma_{\mathrm{T}}}=\frac{\sigma_{\mathrm{T}}}{16 \pi h^{2} B}=0.41,
$$

so that

$$
\Phi_{\mathrm{s}} / \Phi_{0}=0.1508
$$

As a result, if we neglect plural scattering, only $15 \%$ of the flux incident on a "lens" will be useful in scatter focussing.

We will explore four possible configurations of neutron lenses.

\subsection{Neutron Focussing With $A$ A Continuous FUNCTION OF ANGLE}

First, the exact analogue to the multiple Coulomb scattering case of section 2 may be studied, where the atomic mass number $A$ replaces $l$ as the continuous variable to maintain

$$
0_{\mathrm{rms}} \sqrt{2}=r / f \text {. }
$$

We may repeat a measure of the treatment of section 2 by considering a coaxial scattering annulus of length $\lambda$ in the $z$ direction, average radius $r$ and width $d r$, as in fig. 8. A flux $\Phi_{0}$ in the $+z$ direction will result in an incremental flux $\mathrm{d} \Phi$ at $+z=f$ from scattering in the ring given by

$$
\begin{aligned}
\mathrm{d} \Phi & =\Phi_{0} \frac{2 \pi r \mathrm{~d} r}{\mathrm{e} f^{2} \sigma_{\mathrm{T}}} \frac{\mathrm{d} \sigma}{\mathrm{d} \Omega} \\
& =\Phi_{0} \frac{2 \pi p^{2} \sigma_{\mathrm{T}}}{(4 \pi h)^{2} \mathrm{e}} \theta \mathrm{d} \theta \exp \left(-B p^{2} \theta^{2}\right) \\
& =\Phi_{0}\left(3.76 \times 10^{25}\right) p^{2} \sigma_{\mathrm{T}} \theta \mathrm{d} \theta \exp \left(-B p^{2} \theta^{2}\right) .
\end{aligned}
$$

If we define $B^{2}=1 /\left(2 \theta_{0}^{2}\right)$ and use $\sigma_{\mathrm{T}} / B=8 \times 10^{-27} \mathrm{~cm}^{2}$ $(\mathrm{GeV} / c)^{2}$,

$$
\mathrm{d} \Phi=\Phi_{0}(03008) \times \frac{\theta \mathrm{d} \theta}{2 \theta_{0}^{2}} \exp -\left(\theta^{2} / 2 \theta_{0}^{2}\right) .
$$

Now with $A$ adjusted as a function of $r$ so that $\theta=\theta_{0}$, the integrated flux over a range of radii from $r_{1}$ to $r_{2}$ is given by

$$
\Phi_{\mathrm{s}}=\frac{03008}{\mathrm{e}} \Phi_{0} \ln \left(\theta_{2} / \theta_{1}\right)=0.1107 \Phi_{0} \ln \left(\theta_{2} / \theta_{1}\right) .
$$

This ratio $\Phi / \Phi_{0}$ is essentially the same $\Phi / \Phi_{0}$ as found in section 2, eq. (13) reduced by the factor 0.1508 of eq. (25). Unfortunately the range of $\theta_{2} / \theta_{1}$ is also constrained by available materials, since

$$
\begin{aligned}
& \theta_{0}^{2} \propto 1 / B \propto A^{-\frac{2}{3}}, \\
& \theta_{2} / \theta_{1}=\left(A_{1} / A_{2}\right)^{\frac{1}{3}} .
\end{aligned}
$$

A convenient span of materials would range from carbon to lead, where

$$
\left(A_{1} / A_{2}\right)^{\frac{1}{3}}=(208 / 12)^{\frac{1}{3}}=2.58 .
$$

One may do slightly better ranging from beryllıum to uranium, with

$$
\left(A_{1} / A_{2}\right)^{\frac{1}{3}}=(238 / 9)^{\frac{1}{3}}=2.98
$$

The overall enhancement factors are then

$$
\begin{aligned}
& \Phi_{\mathrm{s}} / \Phi_{0}=0.1049, \quad A \text { from } 12 \text { to } 208, \\
& \Phi_{\mathrm{s}} / \Phi_{0}=0.1208, \quad A \text { from } 9 \text { to } 238 .
\end{aligned}
$$

In analogy with eqs. (13) and (19)

$$
\Phi_{\mathrm{T}}=\Phi_{0}\left[1+0.1107 \ln \left(\theta_{2} / \theta_{1}\right)\right],
$$

or

$$
\Phi_{\mathrm{T}} / \Phi_{0} \cong 1.11 \text {. }
$$

This is hardly a spectacular flux improvement.

It is however of greater interest here to consider the spectral response of such a lens. Neutron and neutral kaon beams from a multi-GeV accelerator are generally produced from a beryllium target in a proton beam. For zero degrees production the neutron spectrum is

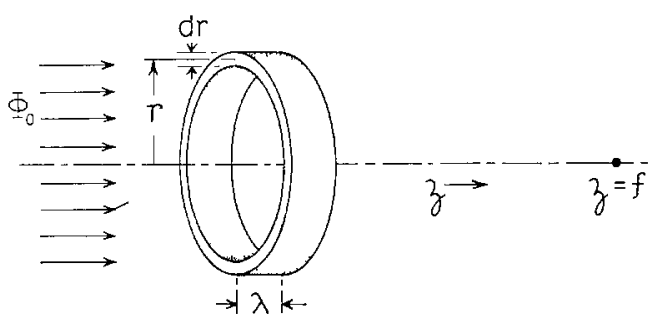

Fig. 8. Neutron scattering annulus of length $l=\lambda$ made of material with atomic number $A$. 
roughly proportional to $p^{2}$ up to some momentum close to the proton momentum, beyond which the spectrum rolls off with a tail related to the Ferm1 motion of nucleons in the target nucleus ${ }^{6}$ ). The momentum spectrum enhancement provided by a diffraction-scattering lens would be exactly as represented in fig. 7 , and by plugging the axis with several mean free paths of material, a neutron beam would be obtained of about $10 \%$ the original intensity and with a spectrum enhanced about a desired momentum. The undesirable aspect of such a lens, in addition to the intensity loss, is the angular divergence of the beam. In many experiments with high-energy neutrons the direction of an incident neutron is a necessary constraint in the data. This is normally quite well known from the event vertex and the neutron-producing target. In typical cases $p \theta$ is of the order of 1 to $0.3 \mathrm{MeV}$ rad. With a carbon scatterer at $\theta_{\mathrm{rms}}, p \theta$ would be $140 \mathrm{MeV}$ rad for a scatter-focussed beam.

It should be noted in both the flux and the spectrum calculations here and below that not only plural elastic scattering but all inelastic scattering has been ignored. It has been our experience in studying total cross sections and small angle elastic scattering of neutrons that the neglect of inelastically scattered neutrons over these small angles characteristic of the diffraction region is very appropriate. Charged particles and $\gamma$-rays are easily and effectively removed from such beams by sweeping magnets and radiators of high- $Z$ materials.

\subsection{The homogeneous plate NeUtron leNS}

We will now consider a different "lens" wherein a slab of a single material one interaction mean free path thick containing a small axial hole is used to enhance the neutron flux as in fig. 9. We will refer to this as the "homogeneous plate" case. In this case, eq. (28) may be integrated with $\theta_{0}$ fixed to give

$\Phi_{\mathrm{s}}=\Phi_{0}(0.150)\left[\exp \left(-\theta_{1}^{2} / 2 \theta_{0}^{2}\right)-\exp \left(-\theta_{2}^{2} / 2 \theta_{0}^{2}\right)\right]$.

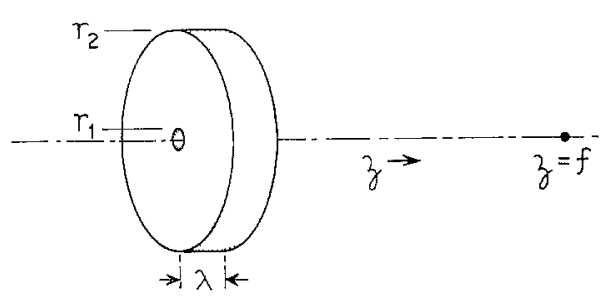

Fig. 9. Homogeneous plate neutron scatterer extending from $r_{1}$ to $r_{2}$ (subtending angles $\theta_{1}$ and $\theta_{2}$ from the focal point).
When $\theta_{1}^{2} \ll \theta_{0}^{2}, \theta_{2}^{2}{ }_{2} \gg \theta_{0}^{2}$, this becomes simply

and

$$
\Phi_{\mathrm{s}} \cong 0.15 \Phi_{0}
$$

$$
\Phi_{\mathrm{T}}=1.15 \Phi_{0}
$$

It seems at first surprising that this factor exceeds the factor 1.1 of eq. (32) for the "optimized" case. The reason, of course, is that the range of $\theta$ here is much greater. On the other hand, no spectral improvement is achieved here.

\subsection{COMBINATION LENS}

The third case which may be explored is one wherein a single, high- $A$ medium is used from a minımum aperture to an intermediate radius, then a graded lens of the first type is used from this radius to the radius corresponding to $\theta_{\mathrm{rms}}$ of the low- $A$ medium, and the low- $A$ medium then extended to a much larger radius In this case the total gain in flux is indeed the sum of the two, i.e.

$$
\Phi_{\mathrm{T}}=\Phi_{0}(1+0.11+0.15)=1.26 \Phi_{0} .
$$

Where maximum flux is the only criterion, this may be an interesting case. The total flux gain, however, seems relatively modest. Such a lens is illustrated diagrammatically in fig. 10.

\subsection{A NEUTRON LENS OF THREE COAXIAL CYLINDERS}

As a final case we may explore a simple and more realistic configuration in lieu of the lens of section 3.2 Nesting cylinders of three materials may be used, with one material of high atomic weight $A_{1}$ extending from $r_{\mathrm{a}}$ to $r_{\mathrm{b}}$, a second of intermediate atomic weight $A_{2}$ from $r_{\mathrm{b}}$ to $r_{\mathrm{c}}$, and a third light material of $A_{3}$ from $r_{\mathrm{c}}$ to $r_{\mathrm{d}}$. Each cylinder would be an interaction mean free path in length, as illustrated in fig. 11. The ratios

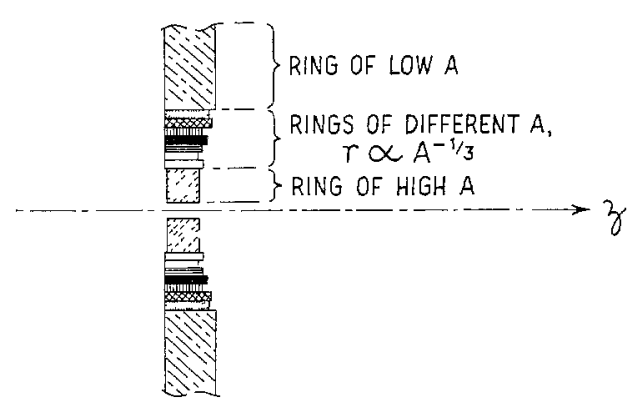

Fig. 10. Schematic section of combination lens with high atomic number medium inside and with low atomic number medium outside a region in which the atomic number is graded according to $r \propto A^{-1 / 3}$. 
of the $r$ 's and $A$ 's may be chosen to yield the same terms in eq. (33) for each material. With angles $\theta$ proportional to radii $r$, we may define intermediate r.m.s. scattering angles $\theta_{1}, \theta_{2}$, and $\theta_{3}$ as

$$
\begin{aligned}
& 2 \theta_{1}^{2}=\left(10 A_{1}^{\frac{2}{3}} p^{2}\right)^{-1}, \\
& 2 \theta_{2}^{2}=\left(10 A_{2}^{\frac{2}{3}} p^{2}\right)^{-1}, \\
& 2 \theta_{3}^{2}=\left(10 A_{3}^{\frac{2}{3}} p^{2}\right)^{-1},
\end{aligned}
$$

so that

$$
A_{1}^{\frac{1}{3}} \theta_{1}=A_{2}^{\frac{1}{3}} \theta_{2}=A_{3}^{\frac{1}{3}} \theta_{3} .
$$

We may set

$$
\theta_{\mathrm{b}} / \theta_{\mathrm{a}}=\theta_{\mathrm{c}} / \theta_{\mathrm{b}}=\theta_{\mathrm{d}} / \theta_{\mathrm{c}}=\theta_{1} / \theta_{2}=\theta_{2} / \theta_{3},
$$

and

$$
\theta_{\mathrm{b}} / \theta_{\mathrm{a}}=\left(2^{\frac{1}{2}} \theta_{1} / \theta_{\mathrm{a}}\right)^{2}, \text { etc. }
$$

This determines $A_{2}$ given $A_{1}$ and $A_{3}$ such that

$$
A_{2}=\left(A_{1} A_{3}\right)^{\frac{1}{2}} \text {. }
$$

The scattered flux $\Phi_{\mathrm{s}}$ at the focal plane of the lens is

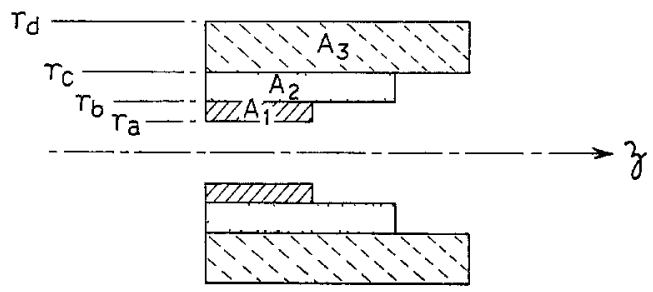

Fig. 11. Schematic section of three-element neutron lens of materials of atomic we1ghts $A_{1}, A_{2}$ and $A_{3}$.

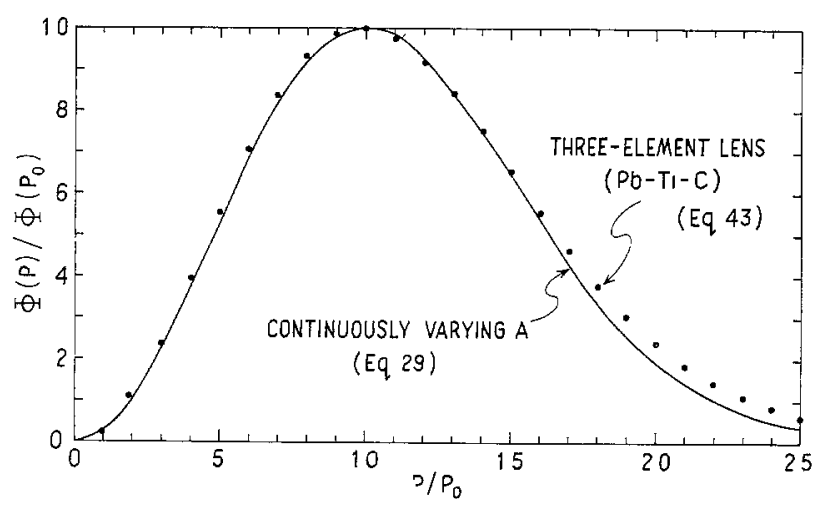

Fig. 12. The spectral response of a three-element neutron lens [eq. (43)], plotted as points, compared with the response of a continuously yarying atomic number lens [eq. (29)]. The smooth curve is Identically that of fig. 7 , and again $\Phi_{0}(p)=\Phi_{0}\left(p_{0}\right)$ is assumed. given in terms of the incident flux $\Phi_{0}$ by

$\Phi_{\mathrm{s}}=\Phi_{0}(3 \times 0.15)\left[\exp \left(-\theta_{\mathrm{a}}^{2} / 2 \theta_{1}^{2}\right)-\exp \left(-\theta_{\mathrm{b}}^{2} / 2 \theta_{1}^{2}\right)\right]$,

and the spectral response will be

$\Phi_{\mathrm{s}}(p)=0.45 \Phi_{0}(p)\left[\exp \left(-p^{2} B_{1} \theta_{\mathrm{a}}^{2}\right)-\exp \left(-p^{2} B_{1} \theta_{\mathrm{b}}^{2}\right)\right]$.

Two numerical examples are explored below. First, with $A_{1}=207.2$ (lead) and $A_{3}=12.01$ (carbon), $A_{2}=49.88$. Titanium, with $A=47.90$ is suitably close. Second, with $A_{1}=238.1$ (uranium) and $A_{3}=9.02$ (beryllum), $A_{2}=46.34$, again close to 47.90 (titanium).

For the $\mathrm{Pb}-\mathrm{Ti}-\mathrm{C}$ lens,

$$
\left(\theta_{\mathrm{a}} / \theta_{\mathrm{b}}\right)=\left(\theta_{\mathrm{b}} / \theta_{\mathrm{c}}\right)=\left(\theta_{\mathrm{c}} / \theta_{\mathrm{d}}\right)=0.621 .
$$

From eq. (37),

$$
\Phi_{\mathrm{s}}=0.45 \Phi_{0}\left[\mathrm{e}^{-0621}-\mathrm{e}^{-1.610}\right]=0.152 \Phi_{0} .
$$

For the U-Ti-Be lerrs,

$$
\left(\theta_{\mathrm{a}} / \theta_{\mathrm{b}}\right)=0.5794, \text { etc. }
$$

and

$$
\Phi_{\mathrm{s}}=0.172 \Phi_{0} .
$$

From eq. (38), the spectra in the two cases are given by, first,

$$
\begin{aligned}
& \Phi_{\mathrm{s}}(p) / \Phi_{\mathrm{s}}\left(p_{0}\right)=\frac{1}{0.33752} \times \\
& \quad \times\left\{\exp \left[-0.621\left(p / p_{0}\right)^{2}\right]-\exp \left[-1.61\left(p / p_{0}\right)^{2}\right]\right\},
\end{aligned}
$$

and, in the second case,

$$
\begin{aligned}
& \Phi(p) / \Phi\left(p_{0}\right)=\frac{1}{0.38224} \times \\
& \times\left\{\exp \left[-0.5794\left(p / p_{0}\right)^{2}\right]-\exp \left[-1.726\left(p / p_{0}\right)^{2}\right]\right\}
\end{aligned}
$$

This spectral response is graphed in fig. 12 together with the spectral response of eq. (29) of section 3.2 (fig. 7). The shape of the response is remarkably similar to that from the structurally impractical continuum of materials.

It may be interesting to consider a numerical example relevant to the new NAL accelerator. Consider a lens optimized for $300 \mathrm{GeV}$ neutrons, with the relationship from eqs. (27) and (24), e.g.

$$
1 / \theta_{\mathrm{a}}=f / r_{\mathrm{a}}=10^{\frac{1}{2}} p_{0} A_{1}^{\frac{1}{3}}\left(\theta_{\mathrm{b}} / \theta_{\mathrm{a}}\right)^{\frac{1}{2}}, \text { etc. }
$$

The parameters for a lens of focal length $f=100 \mathrm{~m}$ are noted in table 1 . 


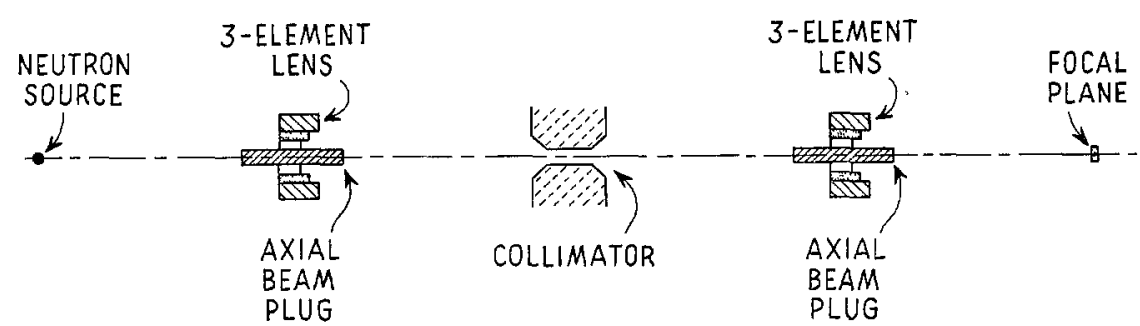

Fig. 13. Schematic section of two-stage neutron beam employing two three-element lenses containing axial beam plugs and a collimator at the first focus.

TABLE 1

Lenses of $100 \mathrm{~m}$ focal length optmized for $p_{0}=300 \mathrm{GeV} / \mathrm{c}$ neutrons.

\begin{tabular}{cccc}
\hline Material & $\begin{array}{c}\text { Length } \\
(\mathrm{cm})\end{array}$ & $\begin{array}{c}\text { Radius } \\
(\mathrm{cm})\end{array}$ & $\Phi_{\mathrm{s}} / \Phi_{0}$ \\
\hline & & 1.404 & \\
$\mathrm{~Pb}$ & 9.5 & 2.260 & 0.152 \\
$\mathrm{Ti}$ & 16.8 & 3.639 & \\
$\mathrm{C}$ & 23.8 & 5.858 & \\
& & 1.294 & 0.172 \\
$\mathrm{U}$ & 6.9 & 2.234 & \\
$\mathrm{Ti}$ & 16.8 & 3.856 & \\
$\mathrm{Be}$ & 24.0 & 6655 & \\
& & & \\
\hline
\end{tabular}

At NAL there is a neutron beam brought to detectors about $400 \mathrm{~m}$ from the neutron production target. By locating such a lens at $200 \mathrm{~m}$ from the target and plugging the central hole with an iron rod absorber of at least $1 \mathrm{~m}$, and placing a collimator of 1 or $2 \mathrm{~cm}$ aperture at $400 \mathrm{~m}$, an enriched flux of about $15 \%$ of the original beam (through that aperture) would be achieved.

\subsection{Two-STAGE NEUTRON FOCUSSING}

It was suggested by Gustafson that in some cases the neutron flux is much greater than needed and that the shape of the spectrum is all important, so that it might be desirable to use a second lens in tandem with the first. The flux at the focus of the second lens would now be about $15 \%$ of the flux incident on the second lens, if it were incident as a parallel beam. In fact, the flux is diverging from the first focus, so that if the second lens is half way between the first and the second foct, the final flux is reduced an additional factor of four. The situation is sketched in fig. 13. The number of neutrons at the second focus, $N_{2}$, is given by

$$
N_{2}=\Phi_{2} A_{2}=\frac{0.152}{4} \Phi_{0}^{\prime} A_{2},
$$

where $\Phi^{\prime}{ }_{0}$ is the fiux incident on the second lens. If $N_{1}$ is the number of neutrons transmitted through the first collimator, $A_{1}$ is the area of the first collimator, and $A_{\mathrm{L}}$ the area of the lens,

$$
N_{1}=\Phi_{\mathrm{s}} A_{1}=\Phi_{0}^{\prime} A_{\mathrm{L}}
$$

so that

$$
N_{2}=\frac{0.152}{4} \Phi_{\mathrm{s}} \frac{A_{1} A_{2}}{A_{\mathrm{L}}}=\frac{(0.152)^{2}}{4} \Phi_{0} \frac{A_{1} A_{2}}{A_{\mathrm{L}}},
$$

or

$$
\Phi_{2}=\left(5.8 \times 10^{-3} A_{1} / A_{\mathrm{L}}\right) \Phi_{0} .
$$

For a $2 \mathrm{~cm}$ diameter aperture $A_{1}$ and the $\mathrm{Pb}-\mathrm{Ti}-\mathrm{C}$ lens of table 1 ,

$$
\Phi_{2}=1.79 \times 10^{-4} \Phi_{0} .
$$

This assumes both lenses contain plugs to block the axial beam, and applies only to the "design" momentum $p_{0}$.

The resulting spectral response, which is acheved at the cost of this loss, is the square of the curve of fig. 12 and is plotted in fig. 14. It appears to us that

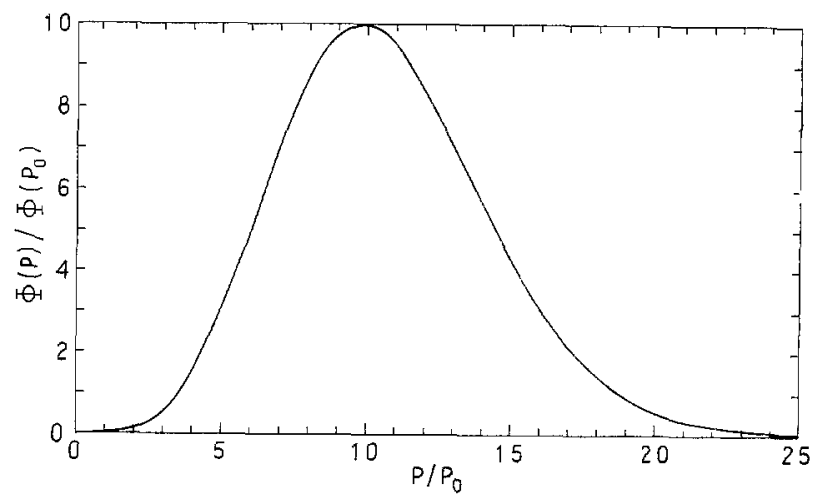

Fig. 14. The spectral response of the two-stage, three-element lens system of fig. 13. The ordinate values are the squares of the corresponding values of fig. 12 . 
only rarely will such a loss in flux justify the spectral response gain.

\section{Conclusions}

We have shown how very simple multiple scattering lenses may provide flux enhancements of charged particles by modest factors $(\times 2-\times 4)$. The practical utilization of such lenses may be very limited except for such special applications as neutrino beams.

Multiple Coulomb scattering also provides the basis for a curious form of radiography using multi-GeV charged particles. Here discontinuties in scattering properties are visualized yielding results somewhat analogous to Xerographic X-rays.

Utilizing similar mathematics, the nuclear elastic scattering of multi-GeV neutrons and other neutral hadrons serves as a mechanism for focussing such particles. The absolute flux gain thus achieved in neutral hadron beams is less than $50 \%$, however the chromatic aberation of such lenses may serve to provide a differential flux enhancement such that a spectrum may be peaked about a desired momentum.

A two-stage lens system is possible, although the greater spectral improvement is only achieved with severe loss of flux.

The authors have profited from ideas and conversa- tions with our colleagues in the University of Michigan high-energy groups. We would like to thank particularly Prof. T. Bowen of the University of Arizona for pointing out an error in the earlier treatment of ref. 1 , and Prof. M. L. Perl of S.L.A.C. for his assistance in the Cosmotron experiments.

\section{References}

1) L. W. Jones, The random optics of particle beams (Univ. of Michigan Technical Report 03106-15-T, 1964) unpublished. L. W. Jones, 12th Intern. Conf. High Energy Physics, vol. 2 (Atomızdat, Moscow, 1966) p. 569.

2) B. Ross1, High energy particles (Prentice Hall, 1952) p. 66. W. H. Barkas and A. H. Rosenfeld, UCRL-8030 TID-4500 (1963) unpublished.

3) W. L. Lakın, E. B. Hughes, L. H. O'Neill, J. N. Otis and L. Madansky, Phys. Lett. 31B (1970) 677.

4) E. F. Parker, T. Dobrowolski, H. R. Gustafson, L. W. Jones, M. J. Longo, F. E. Ring1a and B. Cork, Phys. Lett. 31B (1970) 246.

5) F. E. Ringıa, T. Dobrowolsk1, H. R. Gustafson, L. W. Jones, M. J. Longo, E. F. Parker and B. Cork, Phys. Rev. Lett. 28 (1972) 185.

6) B. G. Gibbard, Thests (Report UM-HE-70-11, Randall Laboratory of Physics, Unversity of Michigan, 1970) unpublished. T. P. McCorriston, Jr., Thesis (Report UM-HE72-11, Randall Laboratory of Physics, Unıversity of Michıgan, 1972) unpublished. D. D. O'Brien, Thesis (Report UM-HE72-32, Randall Laboratory of Physics, Unvversity of Michigan, 1972) unpublished. 